\title{
Reflections on the Unity of Science
}

\author{
Gerald J. Massey \\ University of Pittsburgh
}

Unity of Science. Much fanfare was accorded to the unity of science several decades ago. International congresses met under its banner, an institute was created to promote it, ${ }^{1}$ and an ambitious International Encyclopedia of Unified Science was begun. But interest in the unity of science is hardly of recent vintage. Laplace believed that unity of science had been achieved in principle by 18th century mechanics. And Leibniz's bold conception of a characteristica universalis and scientia generalis was a vision of unitary science. Conceptions of unitary science reach back even into antiquity; the atomism of Democritus and Leucippus and the Pythagorean conception of a world of numbers represent self-conscious, albeit highly speculative, theses of the unity of science.

A once popular thesis maintained that the unity of science consists in the unity of the language of science, specifically in the unity of the observational vocabulary. Herbert Feigl has aptly characterized this thesis as proclaiming the unity of the confirmational basis of the several empirical sciences ([5], p. 227). Feigl's characterization brings out the principal motive behind logical empiricist attempts to circumscribe the so-called observation language. Logical empiricists thought that certain statements (let's agree to call them basic statements) were tied so intimately and overtly to experience that unproblematic observations sufficed to establish their truth values. And they believed that the confirmation or disconfirmation of nonbasic statements, in particular of laws and theories, depended on their logical relations to basic statements. One could recognize the basic statements, so they thought, by means of the kind of descriptive term that occurred in them, the so-called observation term.

No serious philosopher would subscribe today to the thesis of the unity of scientific language so conceived, i.e., as the unity of the confirmational basis. For, as Hilary Putnam has justly remarked, the observational-theoretical dichotomy that underlies this thesis is completely "broken-backed" ([12], p. 241). Putnam does not dispute the need for or utility of an observational-theoretical distinction predicated on the role that statements play in the confirmation or disconfirmation of particular theories. He denies only that the distinction can be drawn on the basis of vocabulary, pointing out that theoretical terms (the distinctive terms of

'The Institute for the Unity of Science, founded by Charles Morris, of which only the name seems to linger on inside the cover of Philosophy of Science. 
a scientific theory) can and do appear in observation statements (the statements used to confirm or disconfirm a theory) ([12], p. 244).

The thesis of the unity of scientific language has assumed other forms. For example, some philosophers have speculated that the descriptive terms of a particular fundamental science suffice to explain the meaning of the descriptive terms of all the other sciences. On can distinguish several versions of this thesis on the basis of the strength of the meaning relation that is supposed to obtain. If the meaning relation is synonymy, then the thesis seems patently false. Surely even the most avid reductionist would stop short of claiming that the term 'temperature of $x$ ' as used in classical thermodynamics is synonymous with 'mean kinetic ernergy of the molecules of $x^{\prime},{ }^{2}$ and such a reductionist would be even less disposed to think that physics (the most popular candidate for the office of the fundamental science) could produce synonyms for such terms as ' $x$ has a headache' or ' $x$ believes that $y$ is $z$ ' (Nagel has suggested that the meaning of a term $t$ of a science $S$ is a function of the $S$-procedures relevant to $t$ and of the $S$-based rules for using $t$ ([10], pp. $297 \mathrm{ff}$. and [11], pp. $352 \mathrm{ff}$.). If this be so, then it would appear impossible that a science whose procedures and rules of usage differ significantly from those of $S$ could supply a synonym for $t$.)

It seems much more reasonable to demand of the fundamental science only that it be able to supply extensional equivalents of the terms of the other sciences. But even this attenuated demand harbors a questionable assumption to which attention is rarely drawn in discussions of unitary science, namely the assumption that the predicates of an arbitrary science are without exception transparent predicates, i.e., predicates all of whose argument places constitute referentially transparent contexts (cf. [9], pp. 228 ff.). But psychology, for instance, seems replete with non-transparent predicates like ' $x$ believes that $y$ is $z$ '. And since the concept of extension applies only to transparent predicates, someone who acknowledges the legitimate presence of non-transparent predicates within certain empirical sciences cannot adopt extensional equivalence as the relevant meaning relation; he must insist upon a stronger relation such as synonymy. Moreover, he must also abandon physics as the fundamental science, at least if he shares the widely entrenched belief that all predicates of physics are transparent, unless he can demonstrate the universal dispensability of non-transparent terms. As late as 1961 Carnap believed that "every nonextensional statement can be translated into a logically equivalent statement of an extensional language" but he was candid enough to call his belief an undemonstrated conjecture ([3], p. ix). Yet in light of the seemingly insuperable obstacles that have blocked attempts to give extensional translations of such sentences as ' $x$ believes that $y$ is $z$ ', Carnpa's

${ }^{2} O f$ course it is possible redefine 'temperature of $x$ ' so that it comes to mean 'mean kinetic energy of $x$, and such redefinition often accompanies a successful reduction. 
conjecture seems not merely undemonstrated but extremely dubious.

Unity of Scientific Language and Unity of Scientific Laws. Still other problems beset extensional equivalence as the meaning relation relevant to the unity of scientific language. There is first the well-known phenomenon that interchange of extensional equivalents sometimes alters the logical status of a sentence. For example, substitution of the extensionally equivalent term 'featherless, tailless biped' for ' $m a n$ ' in the contingent sentence 'Every man is mortal' yields a contingent sentence, whereas the same substitution transforms the contingent truth 'No man has a tail' into a logical truth. Now this phenomenon bears upon a claim of Carnap and others that the unity of scientific laws (in the sense of the derivability of the laws of every science from the laws of the fundamental science together with generalized biconditionals that express the extensional equivalence of the terms of the other sciences with certain terms of the fundamental science) is independent of the unity of scientific language ([2], pp. 60-61). This independence claim rests upon considerations of the following sort.

$\mathrm{L}_{1}$ : Every man is mortal. $\mathrm{L}_{2}$ : All men have backbones.

$\mathrm{L}_{3}$ : No man has a tail. $\quad \mathrm{L}_{4}$ : All featherless, tailless bipeds are mortal. Suppose that $L_{1}, L_{2}$ and $L_{3}$ are laws of a science $S$, and that $L_{4}$ is the sole law of another science $P$ that contains the term 'backbone' but lacks the term 'man'. Let $P^{*}$ be the result of adding to $P$ the generalized biconditional ' $(x)$ ( $x$ is a man if and only if $x$ is a featherless, tailless biped)'. Notice that $\mathrm{L}_{1}$ is derivable within $P^{*}$ because of the fortuitous presence of $\mathrm{L}_{4}$, whereas $L_{2}$ is not derivable within $P^{*}$. So it would appear that whether a particular law of $S$ is derivable in $P^{*}$ depends on what laws happen to be available in $P$, even though $P^{*}$ contains an extensional equivalent for every term of $S$. But consider $\mathrm{L}_{3}$. This law would be derivable in $P^{*}$ regardless of what laws $P$ happened to contain, since $L_{3}$ follows from the generalized biconditional linking 'main' with 'featherless, tailless biped'. If it could be shown that for a certain interesting class $K$ of sciences their laws all behaved much as $L_{3}$ does in the above simpleminded example, it would follow that for sciences in $K$ the unity of scientific language guarantees the unity of scientific laws. And this is precisely what Quine has shown in an important but somewhat neglected paper.

Building upon earlier work of Goodman and himself, Quine has proved the following (cf. [13]). Let $P$ be any first-order theory that includes elementary arithemetic and that contains extensional equivalents for each term of a true theory $S$ that has a finite number of laws and that has a true arithmetical interpretation via constants of elementary arithmetic. Then one can define in $P$ extensional equivalents for the terms of $S$ of such sort that the laws of $S$ all become truths of arithmetic when these co-referential terms are substituted for the terms of $S$. That is, the laws of $S$ are derivable in $P$, by virtue of the generalized bicondi- 
tionals linking the terms of $S$ with their aforementioned defined extensional equivalents in $P$, from truths of elementary arithmetic which, if not already provable in $P$, can be added thereto without modifying the empirical content of $P$. For such sciences, then, the unity of scientific laws is not independent of, but rather guaranteed by, the unity of scientific language. Thus for many theories the thesis of physicalism (the claim that the laws of every science can be derived from the laws of physics) is not, as Carnap thought, a "sweeping extrapolation" but rather a logical consequence of his belief that physics can furnish extensional equivalents for the terms of these theories ([1], p. 883). It is the latter belief, not physicalism, that should be deemed a sweeping extrapolation.

Nagel's Analysis of Reduction: The informal notion of deriving the laws of a science $S$ within another science $P$ is not nearly so clear as it might seem. Nagel calls attention to this unclarity when he discusses the derivability of the BoyleCharles law of classical thermodynamics whithin statistical mechanics. Nagel maintains that derivability of a law $L$ of $S$ within $P$ amounts to the derivability within $P$ of a law that has the same meaning as $L$ ([11], pp. $351 \mathrm{ff}$.). In particular, derivability demands more than derivation within $P$ of a formula iconically similar to $L$, for such a replica of $L$ might be a mere homonym of $L$. As we have already seen, Nagel believes that the meaning of a term of a science $S$ is determined by the procedures and rules of usage of $S$. Hence, for laws of $S$ to be derivable in $P$, the terms of $S$ must somehow be imported into $P$ with their $S$-meanings intact. Therefore, as a necessary condition of the derivability of the laws of $S$ within $P$, Nagel lays down his condition of connectability, to wit: Assumptions must be introduced in $P$ which link the terms that appear in $S$ with the theoretical terms of $P$ ( [11], pp. $353 \mathrm{ff}$.). Nagel envisages only two forms for connecting assumptions, namely generalized biconditionals or generalized conditionals linking primitive $S$-terms with primitive or defined $P$-terms. If the connecting assumptions are all generalized biconditionals, Nagel correctly notes that his condition of derivability (the requirement that the laws of $S$ be derivable from the laws of $P$ with the help of the connecting assumptions and of auxiliary $P$-statements introduced perhaps just to make the derivation possible) is automatically satisfied. The reason is, as Kemeny and Oppenheim have observed, that suitable auxiliary $P$-statements can be obtained by substituting the $P$-terms bidonditionally linked with the $S$-term for the $S$-terms throughout the laws of $S$; the result is a set of auxiliary $P$-statements that, together with the connecting assumptions, entail the laws of $S$ irrespective of what the laws of $P$ happen to be ([8]).

Nagel asserts without proof that satisfaction of his connectability condition does not guarantee satisfaction of his derivability condition ( $[11], p$. 355). The assertion is easily proved; one can show that, given connecting assumptions for all the terms of a theory $S$, it is sometimes impossible to add auxiliary $\boldsymbol{P}$-statements 
so as to make the laws of $S$ derivable from the connecting assumptions, $P$-laws, and auxiliary $\mathrm{P}$-statements without rendering the augmented $P$-etheory inconsistent. ${ }^{3}$ But Nagel seems patently in error when he claims that satisfaction of his derivability condition entails satisfaction of his connectability condition ( $[11], p$. 355). It is trivial to construct examples wherein the laws of a theory $S$ are derivable within a theory $P$ although connecting assumptions of the forms allowed by Nagel (generalized conditionals or biconditionals) are neither present in nor implied by the augmented $P$-theory. To be sure, eccept in the logically trivial cases excluded by Nagel, "connecting assumptions" (sentences containing both $S$-terms and $P$-terms) must be present in the augmented $P$-science, but such "connecting assumptions" have little in common with Nagel's connecting assumptions whose logical forms are strictly circumscribed. So, either Nagel errs in claiming that derivability entails connectability, or he is guilty of equivocating on the notion of a connecting assumption.

Feyerabend's Ccritique of Nagel. Feyerabend has sharply criticized Nagel's analysis of the derivation of the laws of one science within another or, as such derivation is familiary called, of the reduction of one science to another. According to Feyerabend, Nagel's analysis when taken descriptively grossly distorts the important historical episodes of reduction, and when taken normatively Nagel's analysis of reduction is perniciously anti-empiricist ([6], passim). Feyerabend agrees with Nagel that the meaning of a scientific term depends on the theory in which it is embedded, but he puts this doctrine of context-determined meaning to very different use. When two theories differ significantly from one another, Feyerabend contends that at least some of the terms of the theories will be semantically incommensurable ([6], pp. 74 ff.). For example, Einstein's physics differs so significantly from Newton's that Newtonian mass cannot be linked by any connecting assumption to any concept of Einstein's physics because the latter rules out the existence of anything that has the characteristic properties of Newtonian mass. Einstein's laws do not yield Newton's laws as special cases but rather show them to be false. Of course Einstein could show that Newton's laws yield predictions acceptable within certain limits of experimental accuracy for a specifiable range of phenomena and could explain why Newton's laws fail outside these limits. But Feyerabend insists that the foregoing is a far cry from deriving Newton's laws from Einstein's laws supplemented by suitable connecting and auxiliary assumptions. So in the Newton-Einstein reduction and even in Nagel's

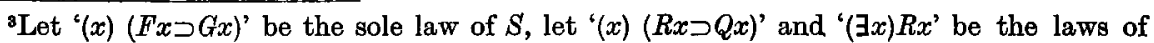
$P$, and let ' $(x)(F x \supset R x)$ ' and ' $(x)(G x \supset Q x)$ ' be the connecting assumptions. Then, where $\Delta$ is any set of $P$-statements, it is easy to show that any model of $\Delta$ that renders the $P$ laws and connecting assumptions true is easily modified to render the $S$-law false without disturbing the truth values of any of the aforementioned formulas. 
example of the reduction of classical thermodynamics to statistical mechanics (here semantical incommensurability is revealed by the fact that, unlike its statistical counterpart, the phenomenological second law admits no entropy decrease in closed systems), Feyerabend avers that Nagel's analysis grossly misrepresents historically noteworthy episodes of reduction.

Let's concede the descriptive inadequacy of Nagel's analysis of reduction and deal now with Feyerabend's charge that, taken normatively, Nagel's analysis is perniciously antiempiricist. A careful reading of Feyerabend reveals that what he considers to be perniciously anti-empiricist is the following maxim: "only such theories are admissible (for explanation and prediction) in a given domain which either contain the theories already used in this domain, or are at least consistent with them" ( $[6]$, p. 44). But Coffa has painstakingly documented his contention that Feyerabend's move from Nagel's model of reduction to the aforementioned anti-empiricist maxim constitutes an egregious non-sequitur that presupposes additional premisses that Feyerabend gratuitously atributes to Nagel, e.g., the absurd idea that it is the goal of science to explain or reduce the existing theories that have proved successful in a given domain ([4]). But to show that Feyerabend's anti-empiricist charge is a non-sequitur is not to show that it is false. Indeed, if Nagel's reduction model is as descriptively defective as Feyerabend maintains, then it would seem quite incredible that it should constitute a healthy norm to guide reductionist attempts. A descriptively more adequate account is called for, but none has been forthcoming. Feyerabend's scattered remarks about reduction hardly amount to an alternative model. Schaffner's formulation of what he styles the Popper-Kuhn-Feyerabend reduction paradigm is not much better suited as a full-blown alternative to Nagel's, because it is vexingly vague and informal at certain critical junctures, and the same is true of Schaffner's own general reduction paradigm ([15]). Like most philosophical business, that of articulating a descriptively adequate (and hopefully normatively fruitful) model of reduction remains unfinished.

Neo-Pythagoreanism. Pythagoreanism was mentioned at the beginning of this paper as an ancient conception of the unity of science. A modern version of Pythagoreanism, which I shall call neo-Pythagoreanism, holds that one is entitled to countenance nothing more than positive integers in bis ontology. Advocates of neo-Pythagoreanism claim that this seemingly absurd doctrine rests upon a celebrated metatheorem of modern logic, the so-called Löwenheim-Skolem theorem. Because there are at least two significantly different metatheorems that could appropriately be called the Löwenheim-Skolem theorem, I will state them both in abbreviated form because their differences will prove relevant to neo-Pythagoreanism: 
$\mathrm{LS}_{1}$ : If a set of first-order formulas has a model (true interpretation) in any nonempty domain, then it has a model in the domain of positive integers.

$\mathrm{LS}_{2}$ : If a set of first-order formulas has a model in a nonempty domain $D$, then it has a model in a countable subdomain of $D$, the terms that occur in the formulas retaining the same meaning in both models. ${ }^{4}$

Neo-Pythagoreanism is an instance of ontological reduction. The motive behind such reduction is ontological economy. To ontologically reduce $S$-objects to $P$ objects is to show that there is no need to countenance $S$-objects over and above $P$-objects. (Nagel was concerned with theoretical reduction, not ontological reduction. Theoretical reduction consists in showing that a theory $P$, appropriately supplemented perhaps, can do the work of another theory $S$ in addition to its own proper work; it aims to explain $S$-objects, but not to explain them away). Familiar examples of ontological reduction are the reduction of ordered tuples to unordered sets, the reduction of positive integers to sets, and the reduction of geometrical points to triples of real numbers. One can applaud these ontological reductions unreservedly, for only abstract objects are eliminated. But indifference to the fate of abstract objects hardly extends to onself. When you and I and the familiar things of daily experience are the candidates for elimination, there is cause for uneasiness if not alarm. Creeping computerization threatens to reduce us to numbers, figuratively speaking. Neo-Pythagoreanism purports to effect that reduction literally.

In a recent article Michael Jubien has argued that the inference from the Löwenheim-Skolem theorem to neo-Pythagoreanism hinges on an additional premiss that I shall call the principle of ontological non-discrimination, to wit: all the models of a theory have equal title to being the theory's ontology ([7], p. 537). Let $S$ be any true theory ostensibly having some familiar ontology. By $\mathrm{LS}_{1}$, there is a model of $S$ in the domain of positive integers. So, by the principle of ontological non-discrimination, one is justified in taking the positive integers as the ontology of $S$, no matter how little resemblance the positive integers bear to the familiar ostensible ontology of $S$. Jubien traces the absurdity of this conclusion to the aburdity of one of its premisses, namely the principle of ontological non-discrimination. He believes that no one would regard a non-standard model of arithmetic (one, for example, that contains elements that cannot be generated from the first element by a finite number of applications of the successor function) to be on equal footing with standard models as candidates for being the ontology of arithmetic ( $[7]$, p. 540). Although the reasons for Jubien's belief are unclear, they seem to presuppose some special intra-theoretical acquaintance with standard models.

'These versions of the Löwenheim-Skolem theorem are stated by van Heijenoort in ([16], p. 253). 
Presumably no one would accept as the ontology of arithmetic a model that is not isomorphic to the standard model of one's special acquaintance. Similarly, Jubien says, the fact that one can prove within standard set theory that there are uncountably many sets suffices to disqualify any countable model of set theory as the ontology of set theory.

Though no better disposed to the principle of ontological non-discrimination than Jubien, I find his reasons against it unconvincing. He seems to suggest that one who understands the theorems of set theory would not be taken in by a countable model thereof. But $\mathrm{LS}_{2}$ knocks the props from under this line of reasoning. $\mathrm{LS}_{2}$ guarantees the existence of a countable model of set theory in which all the sentences of set theory retain their customary meaning, even the theorem which asserts that there are uncountably many sets. Insistence on preservation of meaning is no defense against such radical ontological jerrymandering as trimming an uncountable domain down to countable size. Moreover, since the ontology of any theory can be cut down to denumerable size without disturbing the meaning of any sentences of the theory, the thesis of countable ontologies seems justified, namely "that we are entitled to adopt a countable ontology for any theory we hold" (Jubien calls this thesis, which he rejects, thesis R) ([7], p. 536).

There is but a short step from the thesis of countable ontologies to neoPythagoreanism. It might seem that insistence on meaning preservation prohibits this step; for example, one cannot turn a theory about human beings into a theory about numbers without modifying radically the meaning of its statements. But the trouble with insistence on meaning preservation is that it seems to disqualify all the interesting cases of alleged ontological reduction. Consider two different reductions of ordered pairs to plain sets: in one, $(a, b)$ is identified with $\{a,\{a, b\}\}$; in the other, $(a, b)$ is identified with $\{b,\{a, b\}\}$. On any reasonable criterion of ontological reduction, clearly both of these identifications should qualify. But since the meaning of ' $\{a,\{a, b\}\}$ ' and ' $\{b,\{a, b\}\}$ ' obviously differ, it is impossible that both reductions preserve the meaning of ' $(a, b)$ '. So, it appears that preservation of meaning must not be insisted upon. What, then, might take its place? At first glance, isomorphism looks like a plausible surrogate. That is, one might insist that the system of objects that theory $S$ purports to describe is ontologically reducible to the system of objects that theory $P$ purports to describe just in case the latter system is siomorphic to the former. Unlike insistence on meaning invariance, insistence on isomorphism seems to do justice to the classic examples of ontological reduction. But given the thesis of countable ontologies seemingly justified by $\mathrm{LS}_{2}$, substitution of isomorphism for meaning invariance leads directly to neo-Pythagoreanism. For, given any countable domain together with a set of relations thereupon (i.e., a model structure), there is an isomorphic model structure in the domain of positive integers. 
Several ways to escape neo-Pythagoreanism have been proposed. Quine urges that one recognize ontological reduction only when a proxy function has been specified, i.e., a function that maps in structure-preserving fashion the objects of the theory to be reduced into the objects of the reducing theory ([14], p. 205). Quine notes that the Löwenheim-Skolem theorem does not in general enable one to specify such a proxy function, so neo-Pythagoreanism is averted. But when viewed in the light of the thesis of countable ontologies underwritten by $\mathrm{LS}_{2}$, Quine's appeal to proxy functions seems ad hoc. When the ontology of the theory to be reduced is countable, Quinean proxy functions exist. I fail to see why we should take comfort and encouragement in our not being able to specify proxy functions that we know to exist and that threaten to reduce everything to numbers.

Another suggested escape from neo-Pythagoreanism runs off in another direction; it makes ontological reduction more a matter of the languages of theories than of what the theories purport to be about. Put briefly, it certifies a theory $S$ as ontologically reducible to a theory $P$ just in case there is a term-for-term translation that transforms $S$-theorems into $P$-theorems. The foregoing is essentially Jubien's definition of the conceptual reduction of $S$-objects to $P$-objects ([7], p. 534). It was suggested independently and earlier by Quine as a possible analysis of ontological reduction. Quine rejected it because of certain metamathematical results that cause Jubien to feel uneasy about it, in particular the fact that every axiomatizable theory turns out to be ontologically reducible to arithmetic ([14], p. 202).

Quine reports that the logician G.D.W. Berry once remarked, in view of the Löwenheim-Skolem theorem, that only common sense stands between us and universal Pythagoreanism ([14], p. 201). If so, this state of affairs constitutes a scandal no less serious than Hume's scandal of induction.

\section{References}

[1] Carnap, R., "Herbert Feigl on physicalism," in The Philosophy of Rudolf Carnap, ed. Schilpp, Open Court, La Salle, 1963, pp. 882-886.

[2] Carnap, R., "Logical foundations of the unity of science," International Encyclopedia of Unified Science, vol. I, Univ. of Chicago Press, Chicago, 1955, pp. 42-62.

[3] Carnap, R., The Logical Structure of the World, trans. by R. George, Univ. of California Press, Berkeley, 1967.

[4] Coffa, J., "Feyerabend on explanation and reduction," Journal of Philosophy, vol. 64 (1967), pp. 500-508.

[5] Feigl, H., "Physicalism, unity of science and the foundations of psychology," in The Philosophy of Rudolf Carnap, ed. Schilpp, Open Court, La Salle, 1963, pp. 227267.

[6] Feyerabend, P., "Explanation, reduction, and empricism," Minnesota Studies in the Philosophy of Science, vol. 3 (1962), pp. 28-97.

[7] Jubien, M., "Two kinds of reduction," Journal of Philosophy, vol. 66 (1969), pp. 533541.

[8] Kemeny, J. and Oppenheim, P., "On reduction," Philosophical Studies, vol. 7 (1956), pp. 6-17. 
[9] Massey, G., Understanding Symbolic Logic, Harper and Row, New York, 1970.

[10] Nagel, E., "The meaning of reduction in the natural sciences," in Philosophy of Science, eds. Danto and Morgenbesser, World Publishing Co., Cleveland, 1960, pp. 288-312.

[11] Nagel, E., The Structure of Science, Harcourt, Brace and World, Inc., 1961.

[12] Putnam, H., "What theories are not," in Logic, Methodology, and Philosophy of Science, eds. Nagel, Suppes, and Tarski, Stanford Univ. Press, Stanford, 1962.

[13] Quine, W., "Implicit definition sustained," reprinted in Quine's The Ways of Paradox, Random House, New York, 1966, pp. 195-198.

[14] Quine, W., "Ontological reduction and the world of numbers," reprinted in The Ways of Paradox, pp. 199-214.

[15] Schaffner, K., "Approaches to reduction," Philosophy of Science, vol. 34 (1967), pp. 137-147.

[16] van Heijenoort, J., From Frege to Gödel, Harvard Univ. Press, Cambridge, 1967. 